International Journal of Network Security \& Its Applications (IJNSA), Vol.3, No.3, May 2011

\title{
MAINTAINING UNIFORM DENSITY AND MINIMIZING THE CHANCE OF ERROR IN A LARGE SCALE WSN
}

\author{
Asis Kumar Tripathy ${ }^{1}$,Shradhananda Beura ${ }^{2}$,Muralidhar Behera ${ }^{3}$ and Gunanidhi \\ Pradhan $^{4}$ \\ 1,2,3Department of Computer Science and Engineering, NM Institute of Engineering and Technology, Bhubaneswar,Odisha,India \\ $\left\{{ }^{1}\right.$ asistripathy, ${ }^{2}$ beura. shradhananda, ${ }^{3}$ behera.muralidhar $\}$ dgmail.com \\ ${ }_{4}$ Govt. college of Engineering, Kalahandi, Odisha,India \\ ${ }^{4}$ gunanidhipradhan@gmail.com
}

\begin{abstract}
In a real application area, the WSN is not a homogeneous network where the nodes are maintained in respective coordinate position relatively same to each other. But rather homogeneous it should be heterogeneous, where the relative positional difference for different nodes are different. In this paper a better scheme is being proposed which will take care of the life time and density of a WSN. Sun et. al. proposed uniform density in WSN by assuming the network as a homogeneous network, but in this paper without taking a homogeneous network the same problem is being solved by using the Gaussian probability density function. And also the chance of error in receiving the message from the WSN to the base station is minimized by using priori probability algorithm.
\end{abstract}

\section{KEYWORDS}

WSN, Network density, life time, priori probability

\section{INTRODUCTION}

Sensor networks are composed of small electronic devices, the sensors that monitor areas, objects, animals, persons or sense temperature, humidity, the presence of acoustic or seismic waves etc. in a given area of interest. WSN can be used for remote monitoring and object tracking in different environments and for a wide range of applications. Thus recent advances in computing hardware and software are responsible for the emergence of sensor networks capable of observing the environment, processing the data and making decisions based on the environment, detect and locate specific events and track targets over a specific regions. Thus a sensor network is defined as being composed of a large number of nodes which are deployed densely in close proximity to the phenomenon to be monitored. Each of these nodes collect data and its purpose is to route this information back to a sink. Most previous papers concentrate in homogeneous WSN in many application areas. But practically in many areas homogeneous WSN deployment is not possible. That is the network is to be deployed can be heterogeneous WSN. So, in heterogeneous network the network density over a deployed area may not be uniform. Because in a specified area the position of deployed sensor nodes cannot be maintained in the homogeneous coordinate separation. That is the area covered by the each sensor node's signal is not uniform for the entire deployed sensor node due to the positional 
International Journal of Network Security \& Its Applications (IJNSA), Vol.3, No.3, May 2011

difference is different for every adjacent sensor nodes out of all nodes in WSN. This is one constraint for heterogeneous WSN in real application field.

Another constraint in WSN is the definition of transmitted message from the nodes the sink node, last to the processing device may not be error free due to the attenuation caused by environment in any intentional man made. That is if noise is added to the message during transmission then the message which has been sent from the WSN to the receiver may not be identified as the correct message successfully, due to the addition of noise during transaction period which leads receiving and processing of an erroneous message and the actual sensing event cannot be predicted though it has been censored by the WSN. That's why an algorithm should be developed which can be supported by a specific device and can receive and identify the same message which has been transmitted. This can be done by likelihood probability method called port priori algorithm in which the message received is error free.

\section{RELATED WORK}

Sun et. Al's proposed scheme is based on the homogeneous WSN, where different nodes are deployed with in a predetermined area and the sink node is placed at the coordinate $(0,0)$ of that specified area of the WSN[16]. This paper concentrates on different energy consumption in the network, when the network density is uniform. i.e. the information detected will be forwarded to the sink node so that the energy of the nodes which are closed to the sink will be burnt faster. Once these nodes died, the life time is over the energy of the nodes that are far away from the sink node will be watched, which is inefficient. The waste of energy will be concentrated on this paper to leave nearly no energy when life time comes to its limit, thus makes the network provide service oriented.

The lifetime limits of WSN with and without aggregation have been taken into consideration by Manish Bhardwaj, Anantha P. and Chandrakasan, which gives us the feasibility to do some further work on lifetime[2,4]. After that, a lot of work has been carried out to deal with the lifetime of WSN, the principal goal of which is the efficient use of energy[5-8]. These researches aim at modeling the lifetime and finding a method to optimize it. These work have been done based on the existing definitions of lifetime[9,10]. But on some occasions these standards are inappropriate. The diversity of source behavior and the layout of the network are important factors for they can take effect once the network is deployed[11-14]. Some research work has been done to prolong the lifetime of WSN via unequal clustering or scheduling the selection of cluster heads $[14,15]$. But the solution should be more active, the layout should be well deployed to create wider potential space for optimizing the lifetime before the network takes into action. Different nodes within the network have different service requirements, which inspires the idea of this paper, that is, network lifetime should be service-oriented. A serviceoriented assessment of lifetime is explored in this paper. It is investigated from two perspectives, one is how to assess network lifetime from service-oriented aspect, and the other is how to deploy more nodes to balance the energy consumption where more service is needed.

\section{Over VIEW OF Sun ET. Al's SCHEME}

In this paper there are various definitions are presented. 
definition1: Network density is defined s, in a specified area A where, nodes are deployed, $\rho(x, y)$ is defined as the network density with the property $\iint_{A} \rho(x, y) d x d y=1$ is assumed as homogeneous.

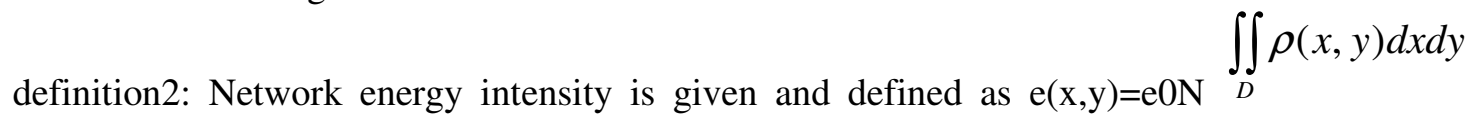
where, $\mathrm{e}(\mathrm{x}, \mathrm{y})$ is defined as network energy intensity with initial energy ${ }_{0}$, for the given region D. With the definition of network density relying models are specified for a routing algorithm $\psi$. The relying probability makes the conditional probability density function $R \psi$ $(\mathrm{x}, \mathrm{y}, \mathrm{a}, \mathrm{b}, \rho(\mathrm{x}, \mathrm{y}))$, with the property $\iint_{A} R_{\psi}(x, y, a, b, \rho(x, y)) d x d y \geq 1$, where $\mathrm{R} \psi$ $(\mathrm{x}, \mathrm{y}, \mathrm{a}, \mathrm{b}, \rho(\mathrm{x}, \mathrm{y}))$ denotes the probability of $(\mathrm{x}, \mathrm{y})$ that will do the rely if there is an event generated at $(a, b)$.The partial density function of the sourced is employed $S(x, y)$ with the property $\iint_{A} S(x, y) d x d y=M$ Where $\mathrm{S}(\mathrm{x}, \mathrm{y})$ denoted the distribution of source behavior with in a period $\mathrm{T}$ and $\mathrm{M}$ denotes the intensity of source behavior.

definition 3: Network requesting intensity $\mathrm{C}(\mathrm{x}, \mathrm{y})$ is defined as :

$\mathrm{C}(\mathrm{x}, \mathrm{y})=\lambda \iint_{A} R_{\psi}(x, y, a, b, \rho(x, y)) S(a, b) d a d b \quad$ Where $\lambda$ is a constant number.

Theorem-1The energy wasted is quantized by the expression.

$$
\iint_{A} C(x, y) d x d y \quad \text { with } \mathrm{e}(\mathrm{x}, \mathrm{y})=\mathrm{T}(\mathrm{C}(\mathrm{x}, \mathrm{y}) \forall(\mathrm{x}, \mathrm{y})) \text { where } \mathrm{T} \text { is a constant value. }
$$

Section 4: Quantization of network density is explained by the density iterative process.

Step 1. i=0; Let the network be uniformly deployed first, then the density distribution $\rho$ is obtained, which is a constant function.

Step 2. After one period $\mathrm{T}$ of data gathering the distribution of energy consumption denoted by network requesting intensity (, ) i c x y is derived.

Step 3. Use (, ) i c x y derived in step 2 to guide the new-round deployment of network density. In section 5, Stability and uniform convergence of the network is analyzed with the equation.

$\rho(x, y)=\frac{\iint_{A} \mathrm{R}_{\psi}(x, y, a, b, \rho(x, y)) S(a, b) d a d b}{\iiint_{A}\left(\int_{A} R_{\psi}(x, y, a, b, \rho(x, y)) S(a, b) d a d b\right) d x d y}$ 
Theorem 2:

It has been explained that.

$$
\begin{gathered}
\iint_{\mathrm{A}} \mathrm{R}_{\psi}\left(x, y, a, b, \rho_{i-1}(x, y)\right) S(a, b) d a d b \\
e_{i}(x, y)=\iiint_{A}\left(\iint_{A} R_{\psi}\left(x, y, a, b, \rho_{i-1}(x, y)\right) S(a, b) d a d b\right) d x d y \\
\text { If } \gamma<1_{\text {by }} \\
\int_{\mathrm{R}}^{\psi} \int_{(\mathrm{x}, \mathrm{y}, \mathrm{a}, \mathrm{b},} R_{\psi}\left(x, y, a, b, \rho_{i}(x, y)\right)=R_{\psi}\left(x, y, a, b, \rho_{0}(x, y)+\eta-\gamma \cdot \rho_{i}(x, y)\right.
\end{gathered}
$$

\section{Theorem-3}

It has been explained that when $\iint_{A} R_{\psi}(x, y, a, b, \rho(x, y)) S(a, b) d a d b$ function then it will dip get uniform convergence.

So over ally, the research on this service oriented network density WSN paper, the network is considered as homogeneous and the density iterative process is prepared to quantize the network density which can enlarge the potential space for optimizing the lifetimes.

Practically when WSN is to be deployed it, can't be homogenous. So in this paper practical approach has been explained where a non-homogeneous i.e heterogeneous WSN can be feasible with the possible error elimination, when message is transmitted. Also it has been proved simulated that, for uniform network the network density $\rho(x, y)$ is with a property $\iint_{A} \rho(x, y) d x d y \neq 1$

\section{Problem Definition}

Section-1

In this scheme, in definition(1), network density is considered and it is denoted as $f(x, y)$. Here as the WSN is considered as homogeneous, therefore the network has taken as uniform network where the network density distribution taken as $\rho(x, y)=\rho_{0}(x, y)$ which is a constant function with a property

$$
\iint_{A} \rho(x, y) d x d y=1
$$


International Journal of Network Security \& Its Applications (IJNSA), Vol.3, No.3, May 2011

For a homogeneous WSN where nodes are deployed uniformly, then the possible schematic diagram of the network will be as follows,

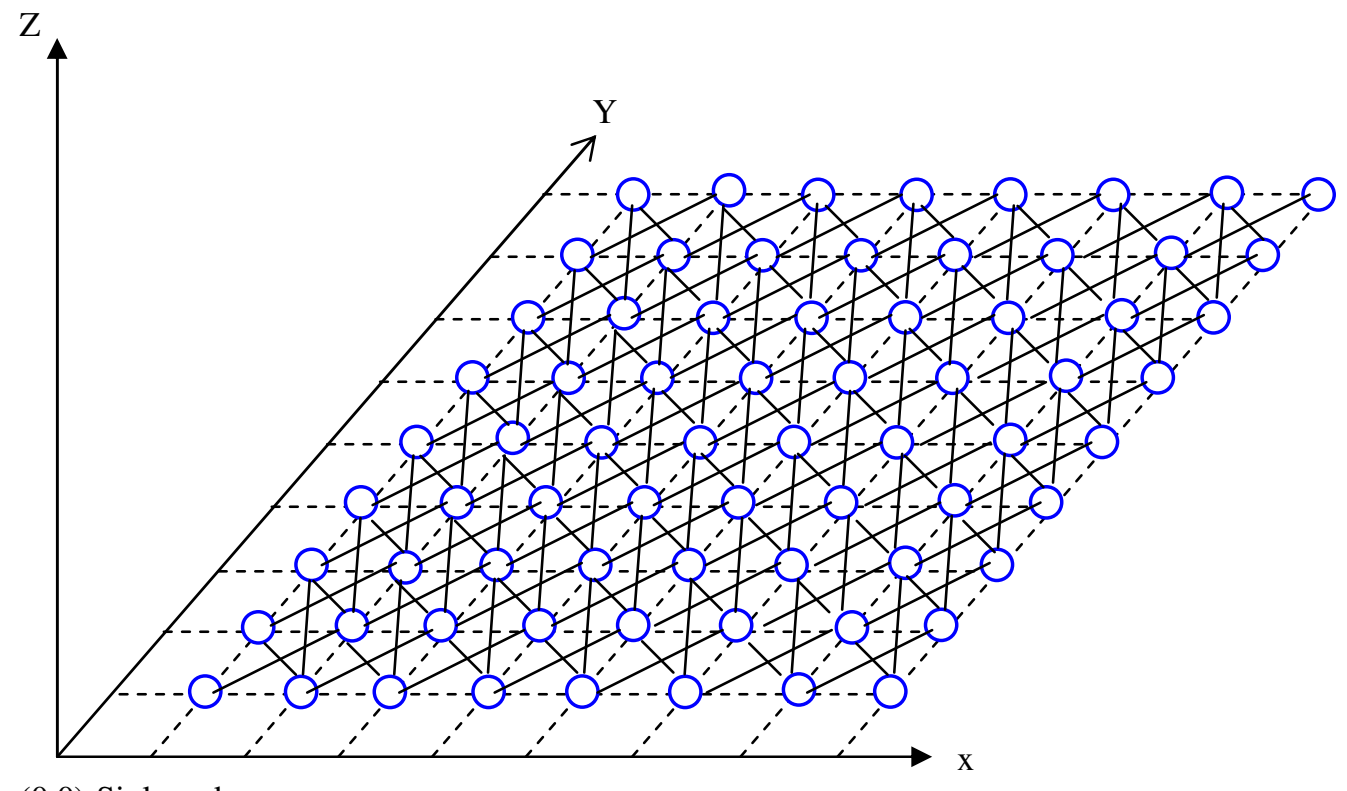

$(0,0)$ Sink node

When the network is uniform i.e the position difference every two consecutive nodes out of all nodes should be same. This is also is represented in the above diagram.

We can assume, the network is uniform if, Positional coordinates $(\mathrm{Nj})$ - Positional coordinates $(\mathrm{Ni})=$ constant $\forall \mathrm{N} \in \mathrm{WSN}$ deployed

$\mathrm{Nj}(\mathrm{x}, \mathrm{y})-\mathrm{Ni}(\mathrm{x}, \mathrm{y})=\mathrm{C} \forall \mathrm{N} \in \mathrm{WSN}$ deployed where, $\mathrm{C}$ is a constant. Suppose the practical area covered by the signals of a specific deployed node inside homogeneous network area is,

$\operatorname{ap}(\operatorname{Nk}(x, y)$ is same for all $\mathrm{n}$ nodes ap

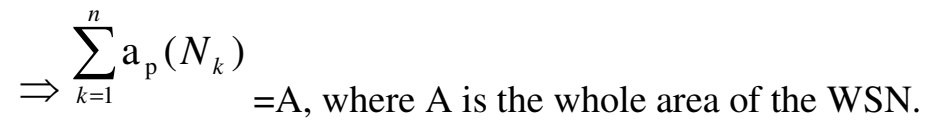

Then for a uniform WSN,

$$
\iint_{A} a_{p}\left(N_{k}\right)(x, y) d x d y=1
$$

But practically a network can not be uniform because when a network is to be established by deploying the nodes over a specified area, then the nodes can not be deployed uniformly over that specified area.

i.e $\mathrm{Nj}(\mathrm{x}, \mathrm{y})-\mathrm{Ni}(\mathrm{x}, \mathrm{y}) \neq \mathrm{C} \forall \mathrm{N} \in \mathrm{WSN}$ deployed 
Practically nodes are deployed non-uniform over a specified area. The likely hood schematic diagram is as follows,

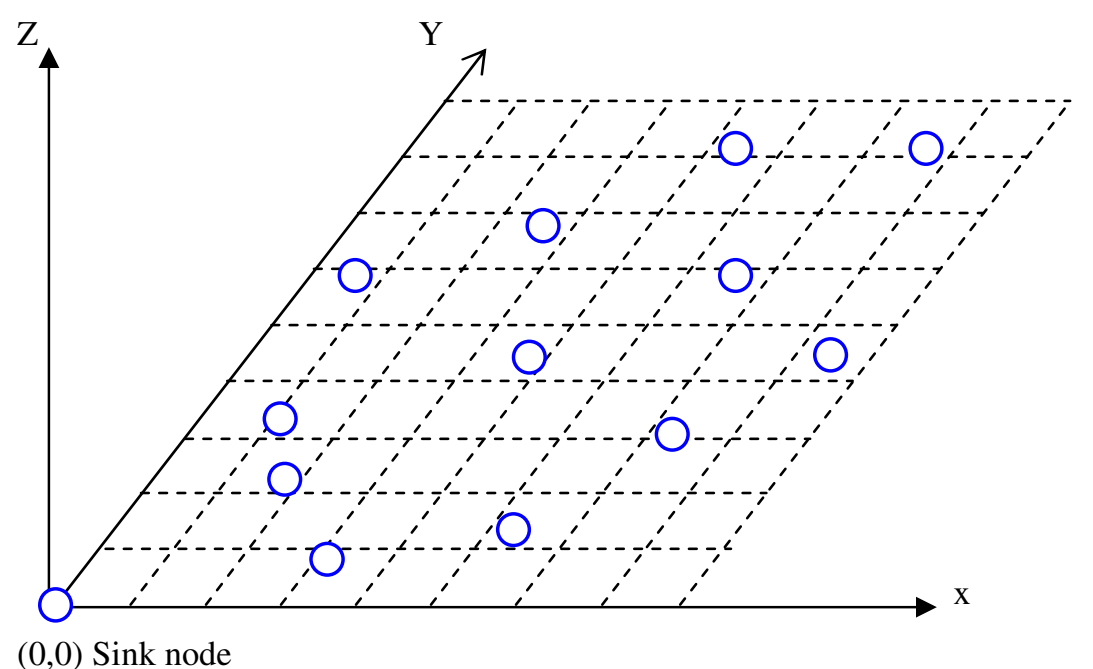

$(0,0)$ Sink node

therefore $\Rightarrow \sum_{k=1}^{n} \mathrm{a}_{\mathrm{p}}\left(N_{k}\right) \neq A$

That means, there exist some partial areas which are not covered by the sensor node; signal. Those are out of coverage of the signal.

Section-2

What happened for the area out of coverage of the signal?

First considering the uniform network, with constant network density $\rho(\mathrm{x}, \mathrm{y})$, there is a routing algorithm $\psi$, the relying probability matches the conditional probability density function $\mathrm{R} \psi$ $(\mathrm{x}, \mathrm{y}, \mathrm{a}, \mathrm{b}, \rho(\mathrm{x}, \mathrm{y}))$, with the property

$$
\iint_{A} R_{\psi}(x, y, a, b, \rho(x, y)) d x d y \geq 1 \text {, where }
$$

$\mathrm{R} \psi(\mathrm{x}, \mathrm{y}, \mathrm{a}, \mathrm{b}, \rho(\mathrm{x}, \mathrm{y}))$ denotes the probability of $(\mathrm{x}, \mathrm{y})$ that will do the relying if there is an event generated at $(a, b)$.

Suppose that event generated at $(a, b)$ is now with in the out of network coverage area of WSN. Then the property of routing algorithm, because the generated event may not be predicted.

i.e. $\iint_{A} R_{\psi}(x, y, a, b, \rho(x, y)) d x d y<1$ 
in theorem 2 it is taken as, $\mathrm{R} \psi(\mathrm{x}, \mathrm{y}, \mathrm{a}, \mathrm{b}, \mathrm{\rho i}(\mathrm{x}, \mathrm{y}))=\mathrm{R} \psi(\mathrm{x}, \mathrm{y}, \mathrm{a}, \mathrm{b}, \rho 0(\mathrm{x}, \mathrm{y}))+\eta_{-} \gamma$. $\mathrm{\rho i}(\mathrm{x}, \mathrm{y})$

where $\iint_{A} \eta-\gamma \cdot \rho_{\mathrm{i}}(x, y) d x d y=0$

where $\rho \mathrm{i}(\mathrm{x}, \mathrm{y})$

$$
=\frac{\iint_{\mathrm{A}} \mathrm{R}_{\psi}\left(x, y, a, b, \rho_{i-1}(x, y)\right) S(a, b) d a d b}{\iiint_{A}\left(\iint_{A} R_{\psi}\left(x, y, a, b, \rho_{i-1}(x, y)\right) S(a, b) d a d b\right) d x d y}
$$

Where $S(a, b) \rightarrow$ the spatial probability density function of a source.

$\rho \mathrm{i}-1(\mathrm{x}, \mathrm{y}) \rightarrow$ the network density distribution of the preceding node $\mathrm{Ni}$ and this $\rho \mathrm{i}(\mathrm{x}, \mathrm{y})$ converges if $\gamma<1$.

But for a non-uniform network, it may not be converged for $\gamma<1$, because $\gamma<1$

$$
\iint_{A} R_{\psi}(x, y, a, b, \rho(x, y)) d x d y<1
$$

For uniform network the network density is $\rho(\mathrm{x}, \mathrm{y})$ with a property $\iint_{A} \rho(x, y) d x d y=1$ practical non-uniform network, using Gussian probability density we can perform a number of experiments to verify that

$$
\iint_{A} \rho(x, y) d x d y \neq 1
$$

The Gaussian probability density function is $\mathrm{f}(\mathrm{x})=\frac{1}{\sqrt{2 \pi \delta^{2}}} \mathrm{e}^{-(x-m)^{2} / 2 \delta^{2}}$

Where, $\mathrm{m}$ and $\delta^{2}$ are the average value and the variance associated with Gaussian probability density function $\mathrm{f}(\mathrm{x})$.

Thus $\bar{X}=\int_{-\infty}^{\infty} \frac{x e^{-(x-m)^{2} / 2 \delta^{2}}}{\sqrt{2 \pi \delta^{2}}} d x=\mathrm{m}$ 
Now the cumulative distribution corresponding to the Gaussian probability density for $m=0$, is $\mathrm{p}(\mathrm{X} \leq \mathrm{x})=\mathrm{F}(\mathrm{x})=\int_{-\infty}^{x} \frac{x e^{-x^{2} / 2 \delta^{2}}}{\sqrt{2 \pi \delta^{2}}} d x$

Now, the error function of $\mathrm{u}$ is given by,

$\operatorname{erf} \mathrm{u}=\frac{2}{\sqrt{\pi}} \int_{0}^{u} e^{-u^{2}} d u$

the complementary error function is denoted as erfc $\mathrm{u}$ and given by,

erfc $u=1$-erf $u$

$=\frac{2}{\sqrt{\pi}} \int_{0}^{\infty} e^{-u^{2}} d u$

Now the cumulative distribution function $F(x)$ is expressed in terms of the error function and the complementary function, for $\mathrm{x} \geq 0$,

$$
\begin{aligned}
\mathrm{F}(\mathrm{x}) & =\int_{-\infty}^{x} \frac{x e^{-x^{2} / 2 \delta^{2}}}{\sqrt{2 \pi \delta^{2}}} d x \\
& =\int_{-\infty}^{\infty} \frac{x e^{-x^{2} / 2 \delta^{2}}}{\sqrt{2 \pi \delta^{2}}} d x \int_{-x}^{\infty} \frac{x e^{-x^{2} / 2 \delta^{2}}}{\sqrt{2 \pi \delta^{2}}} d x
\end{aligned}
$$

For $\mathrm{x} \leq 0$, for positive $\mathrm{u}$,

$\mathrm{F}(\mathrm{x})=\mathrm{F}(-|\mathrm{x}|)=\int_{-\infty}^{-|x|} \frac{x e^{-x^{2} / 2 \delta^{2}}}{\sqrt{2 \pi \delta^{2}}} d x=\frac{1}{\sqrt{\pi}} \int_{-\infty}^{-|x| / \sqrt{2} \pi} e^{-u^{2}} d u$

Let $\varepsilon=-u$

$\mathrm{F}(\mathrm{x})=\frac{1}{2}\left(\frac{2}{\sqrt{\pi}} \int_{|x| / \sqrt{2} \delta}^{\infty} e^{-\varepsilon^{2}} d \varepsilon\right)=\frac{1}{2} \operatorname{erfc}\left(\frac{|x|}{\sqrt{2} \delta}\right)$ 
Now, for a constant number k, the probability on the standard deviation $\delta$,

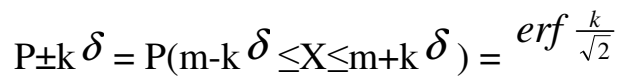

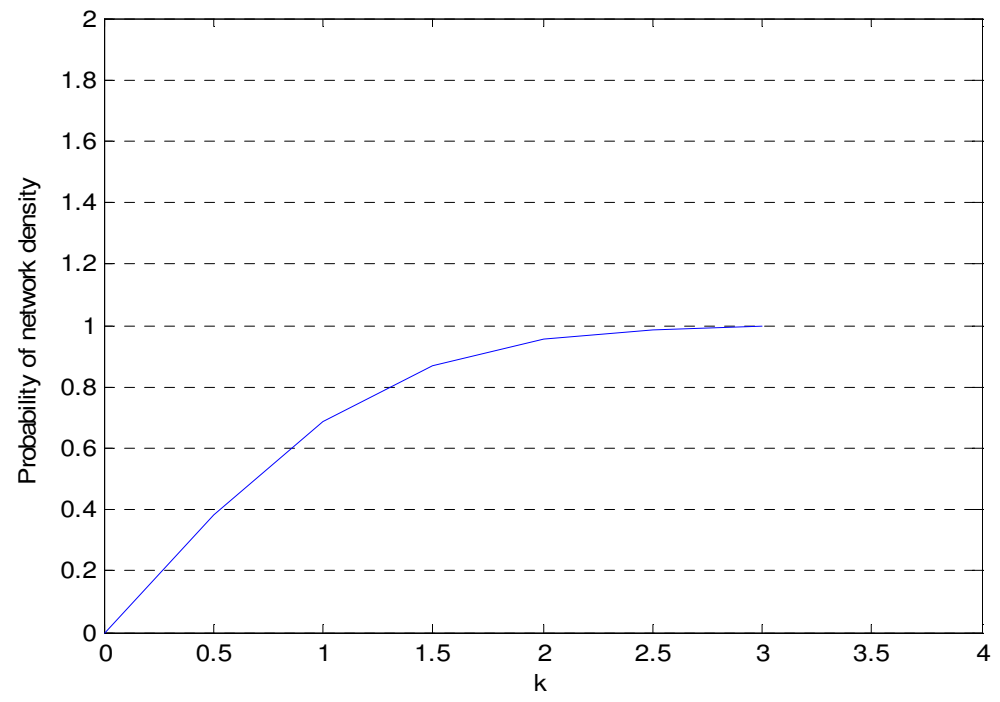

Section-3

Error calculation:

As the signal traverses the transmission medium noise will be added to the signal. Hence as finally received and pre received the signal will be corrupted or contaminated by noise from a number of sources.

Let us consider one case:

In successive intervals we want to transmit one of the two possible messages. The two possible messages might be represented at the transmitting end by two distinct wave form, each limited in time period to the interval allocated to a bit. At the receiving end we right desire a system where by the message $\mathrm{m} 0$ when received, generates some voltage $\mathrm{r} 0$, for $\mathrm{m} 1$ received generates voltage $\mathrm{r} 1$ in the absence of noise. Because of noise, error occurred i.e for message $\mathrm{m} 0$ received indication might be $\mathrm{r} 1$ and for $\mathrm{m} 1 \rightarrow \mathrm{r} 0$

We shall assume, for generality, that the probability of an error is dependant on which msg was send and transition probabilities are introduced. i.e

$\mathrm{P}(\mathrm{r} 0 \mid \mathrm{m} 0)=$ probability that $\mathrm{r} 0$ is received for $\mathrm{m} 0$ sent.

$\mathrm{P}(\mathrm{r} 1 \mid \mathrm{m} 0)=$ probability that $\mathrm{r} 1$ is received for $\mathrm{m} 0$ sent.

$\mathrm{P}(\mathrm{r} 0 \mid \mathrm{m} 1)=$ probability that $\mathrm{r} 0$ is received for $\mathrm{m} 1$ sent. 
$\mathrm{P}(\mathrm{r} 1 \mid \mathrm{m} 1)=$ probability that $\mathrm{r} 1$ is received for $\mathrm{m} 1$ sent.

\section{Priori probabilities:}

For the general case, messages $\mathrm{m} 1$ and $\mathrm{m} 0$ do not occur with equal frequency and probabilities $\mathrm{p}(\mathrm{m} 1)$ and $\mathrm{p}(\mathrm{m} 0)$ are introduced. Probabilities that $\mathrm{m} 1$ and $\mathrm{m} 0$ intended in an arbitrary message interval. These probabilities $\mathrm{p}(\mathrm{m} 1)$ and $\mathrm{p}(\mathrm{m} 0)$ are called priori probabilities.

Now from an observed response $\mathrm{r} 1$ and $\mathrm{r} 0$, it can not be determined with certainty which of the message was sent. Now by posteriori probability (message intended with maximum probability that our opinion is correct).

If $\mathrm{r} 0$ is received

Choose $\mathrm{m} 0$ if $\mathrm{P}(\mathrm{m} 0 \mid \mathrm{r} 0)>\mathrm{P}(\mathrm{m} 1 \mid \mathrm{r} 0)$

Choose $\mathrm{m} 0$ if $\mathrm{P}(\mathrm{m} 1 \mid \mathrm{r} 0)>\mathrm{P}(\mathrm{m} 0 \mid \mathrm{r} 0)$

If $\mathrm{r} 1$ is received

Choose $\mathrm{m} 1$ if $\mathrm{P}(\mathrm{m} 0 \mid \mathrm{r} 1)>\mathrm{P}(\mathrm{m} 1 \mid \mathrm{r} 1)$

Choose $\mathrm{m} 1$ if $\mathrm{P}(\mathrm{m} 1 \mid \mathrm{r} 1)>\mathrm{P}(\mathrm{m} 0 \mid \mathrm{r} 1)$

Similarly,

If $\mathrm{r} 0$ is received, $\mathrm{m} 0$ should be chosen if

$\mathrm{P}(\mathrm{m} 0 \mid \mathrm{r} 0) \mathrm{P}(\mathrm{r} 0)>\mathrm{P}(\mathrm{m} 1 \mid \mathrm{r} 0) \mathrm{P}(\mathrm{r} 0)$

Correspondingly If $\mathrm{r} 1$ is received, $\mathrm{m} 1$ should be chosen if

$\mathrm{P}(\mathrm{r} 1 \mid \mathrm{m} 1) \mathrm{P}(\mathrm{m} 1)>\mathrm{P}(\mathrm{r} 1 \mid \mathrm{m} 0) \mathrm{P}(\mathrm{m} 0)$

This is optimum - receiver algorithm.

In general, there can be $\mathrm{k}$ messages $\mathrm{m} 1, \mathrm{~m} 2, \ldots \ldots . \mathrm{mk}$ and $\mathrm{j}$ received rensposes $\mathrm{r} 1, \mathrm{r} 2, \ldots \ldots \ldots \mathrm{rj}$, by optimum-receiver algorithm, if $\mathrm{rj}$ is received, choose $\mathrm{mk}$ if

$\mathrm{P}(\mathrm{mk} \mid \mathrm{rj})>\mathrm{P}(\mathrm{mi} \mid \mathrm{rj}) \forall \mathrm{i \neq k}$

Optimum receiver:

A receiver which operates in accordance with this algorithm to maximize a posteriori probability of a correct decision and is called an optimum receiver. 


\section{CONCLUSION}

In this paper, it is more concentrated on the density of the network in a heterogeneous WSN. A method to assess lifetime from the perspective of service is first presented, in which whether the network can support service is concentrated on and also the chance of error at the receiving site is strictly minimized. This is also being analyzed that how the sink node will be used for maximum time then also there will not be any problem because of the lifetime of the sensors. Deployment can be guided to enlarge the potential space for optimizing the lifetime of the network. The Proposed method is also practically being analyzed the feasibility of the network.

\section{REFERENCES}

[1] Akyildiz I.F, Su W, etc. Wireless sensor network: A survey [J]. Computer Networks. 2002, 38(4): 393-422.

[2] Bhardwaj M, Chandrakasan A, Garnett T. Upper bounds on the lifetime of sensor networks[C]. In: IEEE Int'1 Conf. on Communications. Helsinki: IEEE Computer Society, 2001, 785-790.

[3] Duarte-Melo E. J, Liu M, Misra A. A Modeling Framework for Computing Lifetime and Information Capacity in Wireless Sensor Networks[C]. In: Modeling and Optimization in Mobile, Ad Hoc and Wireless Networks, Cambridge, UK, March 2004.

[4] Bhardwaj M, Chandrakasan A.P. Bounding the Lifetime of Sensor Networks Via Optimal Role Assignments [C]. In: INFOCOM. New York, USA: IEEE, 2002. 1587-1596.

[5] Kalpakis K. Efficient Algorithms for Maximum Lifetime Data Gathering and Aggregation in Wireless Sensor Networks [J]. Computer Networks: The International Journal of Computer and Telecommunications Networking. August 2003, 42(6): 697-716.

[6] Enrique J. Duarte-Melo, Mingyan Liu. Analysis of Energy Consumption and Lifetime of Heterogeneous Wireless Sensor Networks[C]. In: Proc. of the GLOBECOM 2002. New York: IEEE Press, 2002. 21-25.

[7] Rai V, Mahapatra R. N. Lifetime Modeling of a Sensor Network[C]. In: Design , Automation and Test in Europe, Munich , Germany ,March 2005. 202-203.

[8] Yuan Xue, YI CUI, etc. Maximizing Lifetime for Data Aggregation in Wireless Sensor Networks[J]. Mobile Networks and Applications. 2006, 10(6): 853-864.

[9] A. Ephremides. Energy Concerns in Wireless Networks [J]. IEEE Wireless Communications, vol. 9, pp. 48-59, Aug. 2002.

[10] Jianping Pany Y. Thomas Houz Lin Caiy etc. Topology Control for Wireless Sensor Networks [C]. In: nternational Conference on Mobile Computing and Networking. 2003, 286-299.

[11] Egorova-Forster, Murphy. Exploring Non Uniform Quality of Service for Extending WSN Lifetime[C]. In: Fifth IEEE International Conference on Pervasive Computing and Communications Workshops (PerComW'07) 2007. 285-289.

[12] Lu Ke zhong, Huang Liu sheng, etc. Deploying Sensor Nodes in Wireless Sensor Networks[J]. Journal of Chinese Computer Systems. 2006, 27(11):2003-2006.

[13] Guihai Chen. Chengfa Li. An Uneven Cluster-Based Routing Protocol for Wireless Sensor Networks [J]. Chinese Journal of Computers. 2007, 30(1):27-36 
International Journal of Network Security \& Its Applications (IJNSA), Vol.3, No.3, May 2011

[14] Soro S, Heinzelman WB. Prolonging the lifetime of wireless sensor networks via unequal clustering[C]. In: Proc. of the 19th IEEE Int'l on Parallel and Distributed Processing Symposium. San Francisco: IEEE Computer Society Press, 2005. 236-240.

[15] Biswanath Dey Sukumar Nandi. Distributed Location and Lifetime Biased Clustering for Large Scale Wireless Sensor Network[C]. In : Distributed Computing and Networking, $8^{\text {th }}$ International Conference, ICDCN 2006. 534-54

[16] Dayang Sun,Yanheng liu,Aimin wang, Bin Ge Research on Service-oriented Lifetime and Network Density in WSN In:IEEE 9th International Conference for Young Computer Scientists 2008, 439-444

[17] Communication System, Taub \& Sheiling.

\section{Authors}

Asis Kumar Tripathy Received Diploma in IT in 2002, B.E. IT in 2006 and MTech in Comp. Sc. \& Engg. In 2010 from Inter-National Institute of Information Technology, Bhubaneswar. He has Joined NM Institute of Engg. \& Technology as a lecturer from 01-07-2010.His research areas are Network Security and Wireless Sensor Network.

Shradhananda Beura received Diplma in IT, BTech. From CET, Bhubaneswar In 2004 \& 2008 respectively. He is persuing MTech. In CSE. His research area is Wireless Sensor Network.

Muralidhar Behera received MCA in 2003, Persuing MTech. In CSE. His research Sensor Network.
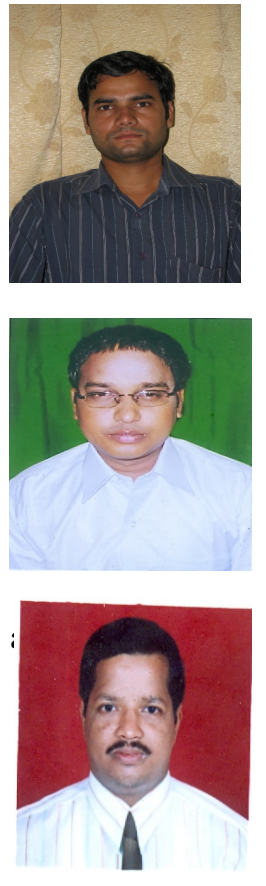

Dr. Gunanidhi Pradhan Received Ph.D in Information \& Communication Technology.

Awarded Best Teacher Award by ISTE,Currently Principal, Govt. College of Engineering, Kalahandi,Bhawanipatna,Odisha

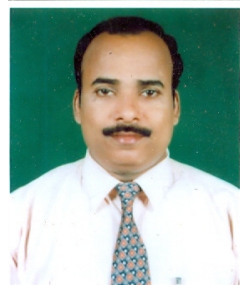

